\title{
Frontières
}

\section{La mort jouée}

\section{Régine Bruneau-Suhas}

Volume 19, numéro 2, printemps 2007

\section{Penser sa mort?}

URI : https://id.erudit.org/iderudit/017499ar

DOI : https://doi.org/10.7202/017499ar

Aller au sommaire du numéro

\section{Éditeur(s)}

Université du Québec à Montréal

ISSN

1180-3479 (imprimé)

1916-0976 (numérique)

Découvrir la revue

\section{Citer cet article}

Bruneau-Suhas, R. (2007). La mort jouée. Frontières, 19(2), 59-61.

https://doi.org/10.7202/017499ar

Ce document est protégé par la loi sur le droit d'auteur. L'utilisation des services d'Érudit (y compris la reproduction) est assujettie à sa politique d'utilisation que vous pouvez consulter en ligne.

https://apropos.erudit.org/fr/usagers/politique-dutilisation/
Cet article est diffusé et préservé par Érudit.

Érudit est un consortium interuniversitaire sans but lucratif composé de l’Université de Montréal, l'Université Laval et l'Université du Québec à Montréal. Il a pour mission la promotion et la valorisation de la recherche. https://www.erudit.org/fr/ 


\section{LA MORT JOUÉE}

rible et le macabre. Mon corps de pierre apparaît figé, totalement immobilisé, et en même temps semble-t-il s'affaisser sur soi, refusant toute rigidité qui le qualifierait de mort. Ariès (1985) analyse ce qu'est un Gisant et oriente l'interprétation que je peux en donner. Le Gisant appartient au monument funéraire d'une ancienne liturgie, celle des Dormants, des Reposants. Ce ne sont ni des vivants insouciants, ni des agonisants sur un lit de douleur, ni des morts destinés à la putréfaction ni non plus des ressuscités dans la gloire, mais des élus qui attendent dans le repos et la paix la transfiguration du dernier jour, la résurrection. Le Gisant est reconnaissable, c'est un beati, un bienheureux (Ariès, 1985, p. 238). C'est à partir du XIVe siècle que les yeux du Gisant se ferment. Le Gisant se trouve, au commencement, dans la position tout à fait invraisemblable de la statue debout-couchée, debout par les plis non tombants de ses vêtements et la retenue de ses membres, défiant alors toutes les lois de la pesanteur. Cette persistance à vouloir disposer le mort dans une position de repos tout en suggérant en lui le vivant lui confère un grand sentiment de paix éternelle. Le Gisant sera bientôt dressé, voire il s'inscrira en une posture animée, soit assis, soit agenouillé. Ariès décrit ainsi les dernières traces de l'ancienne attitude de ce qu'il nomme «la mort apprivoisée»: «S'en aller, mais pas pour toujours, seulement pour dormir longtemps, mais d'un sommeil qui laisse les yeux ouverts, qui ressemble à la vie sans être tout à fait la vie, ni la survie» (1985, p. 239). Le Gisant donne donc une représentation réaliste de la mort tout en conservant une forme illusoire de la vie. Le Gisant ne s'éloigne guère de son modèle originel. Le Gisant se tient au plus proche de son double celui qui gît dessous, le corps corruptible inclus à la terre. Le Gisant est à proximité d'un état neutre qui le fait s'éloigner soit vers la mort, soit vers la vie, soit vers la béatitude. Cet état neutre transmet l'idée ancienne d'un intermédiaire sis par-delà la mort qui sied bien à la pensée de ma propre mort, moi qui veux jouer à être morte sans jamais l'être totalement.

\section{ENTRE RÉEL ET IRRÉEL: APPARENCE}

L'antithèse de la Mort de Soi costumée en Gisant s'offre au monde en une figuration baroque; elle se tient au bord de l'abîme, immobile et silencieuse, incarnée dans une sorte de pierre. Elle livre le monde des apparences, la figure infigurable de l'absence, le regard plongé en un face à face éternel avec l'humain transfiguré. Elle diffuse cet écart entre l'humain et l'inhumain, à l'interface d'une mort sculptée et jouée qui n'est pas même la mort; elle apparaît encore dans la transparence d'un absent en même temps que dans la présence d'un corps artistique pensé, moi-même qui en tant que figure théâtrale joue sa propre mort. L'utopie d'une transparente image concourt à la présentation d'un réel par l'exposition d'un corps d'humain mort, et à la suggestion de l'irréel par l'idéal d'une parenté avec les restes de ma propre vie qui s'en dégageraient. La figure de la Mort de Soi telle qu'elle apparaît sur le site théâtral cimetiéral entretient une opposition entre le tombal de la matérialité d'une vie qui serait finie, consommée et l'idéal d'une mort d'apparence, d'une mort jouée, sise entre la corporéité de l'humain et l'évanescence de l'inhumain, voire d'un dieu immortel, entre la réalité de la sculpture et la virtualité de son incarnation, entre l'état moderne d'une défigure profane, ou profanée et l'archaïsme d'une figure sacrée. La figure de la Mort de Soi, telle que je 
la joue sur scène, fait et défait des vies imaginaires, toujours se tenant à distance de son propre corps tout en ajointant la présence latente de l'impalpabilité de la mort. La mort est là, pressentie, comme l'idée de ce qui peut un jour advenir ou de ce qui est advenu sans vraiment l'être. Le silence eschatologique dont elle s'entoure nourrit l'âpreté du devenir mortel de cette humanité.

\section{UNE QUÊTE DE L'INVISIBLE}

L'espace de la mort, la scène comme cimetière va permettre de comprendre la manière dont se manifeste le monde d'un «faire comme si » de ma propre mort jouée et de s'approcher des choses et des lieux tels qu'ils se présentent naturellement au sein d'un monde défunt. Le cimetière, le cigît comme espace théâtral, s'expose, investi de l'insolite et du pittoresque de la mort. L'espace de la mort en tant que tel entre en scène et se fait paysage. Mon cimetière figure la mort, un univers semi-englouti dans un plancher scénique en poussière. Le cimetière sert bien entendu à enterrer le mort, à l'éloigner de la vie et offre ici l'esthétique d'une sublime désolation. La nécropole théâtrale étale le spectacle d'un laisser-là, d'une architecture funéraire disloquée, n'ayant en soi aucun désir de produire une quelconque allusion à la vie, tout abandonnée à la fouille du théâtrologue avide de la pensée de sa propre mort. Elle est un espace indifférencié au bord du vide, absolument confondu à ce qui reste indiscutable. La forteresse théâtrale des morts est isolée en zone "interdite aux promeneurs». Elle signale seulement le lieu. Comme un grand cercueil d'artifice, la nécropole théâtrale n'est plus qu'un lieu de traversée, un lieu d'exploration, de gisement. C'est là que la pensée de ma propre mort prend sa fondation.

La grande scène du monde, une fois travestie en sépulcre, entre en médiation mais aussi en méditation avec la pensée de la mort et veut négocier le secret, si secret il y a. Secrète, cette tombe l'est; elle garde un silence millénaire. Il s'agit, ici, d'un lieu travesti de mes restes offerts en simulacre, dans une extrême confusion de territoire où l'espace de la mort ne cache rien à sa surface puisqu'il présente le Gisant, l'image sculpturale de ma propre mort jouée. Le lieu exposé en totale instabilité spatiale et temporelle est la manifestation apparente d'un monde défait, celui de la mort. Pourtant, un seuil tente d'être franchi. Des effluves de rien, de destruction viennent caractériser l'ineffable qui émane des fragments de matière, de pierre et d'humain. Ces restes-là, ce qui gît sous l'image du Gisant, rien ne les rassemble à un désir de monde à habiter et où séjourner, si ce

LE GISANT SE TROUVE DANS LA POSITION TOUT À FAIT INVRAISEMBLABLE

DE LA STATUE DEBOUT-COUCHÉE, DEBOUT PAR LES PLIS NON TOMBANTS

DE SES VÊTEMENTS ET LA RETENUE DE SES MEMBRES,

DÉFIANT ALORS TOUTES LES LOIS DE LA PESANTEUR.

n'est pour l'éternité. C'est pourtant à partir de là qu'il faut poser l'ancre par le fond et trouver une fondation pour penser le sujet vif de la Mort de Soi, distancée de mon propre moi.

C'est ainsi que le Gisant profile l'image défunte d'une ombre de la Mort de Soi. Elle se projette au sol dans l'aube d'un monde nouveau, finit par dissocier la tragédie et pose le jeu en une magistrale re-présentation d'un sujet vif obscurci par toutes les implosions qui l'habitent. L'ombre déplace le sujet de toute l'agitation mortuaire qui finit par l'habiter et l'inscrit dans une transfiguration. L'anamorphose joue avec les apparences de mort dans la vie. Elle part à la recherche d'un ordre définitivement dessaisi. L'ombre de ma propre mort, en une Mort de Soi costumée, glisse sur le dos bombé du tumulus théâtral, recréant au sol le précieux nœud d'une sorte de croisement vital. La confrontation en surface a lieu entre l'espace théâtral néantisé par la mort et le contour précaire de l'ombre, elle aussi projetée dans la présentation de la Mort de Soi. Mon ombre se lie à cette mort jouée et figurée en Gisant de telle sorte qu'elle hante le monde des vivants car un être sans ombre n'est pas. Elle délivre le sujet vif de son corps de carton-pâte, le travestit en corps de l'ombre et l'inscrit dans la matière spectrale et cimetiérale dont le théâtre aime se nourrir. L'ombre est le contraire de la lumière et lui fait obstacle. Elle s'imprime à la surface en un contour qui coupe, découpe le corps, en conserve la trace et empêche l'effacement. C'est ainsi qu'en un retournement, mon visage et mon corps d'ombre, le tout imprimé contre terre, s'inscrivent en négatif sur le blanc scénique d'un espace défunt. La réalité bascule dans une reproduction inversée du sujet. Le sens du mot envers «est d'abord employé pour désigner le côté normalement invisible, opposé à l'endroit, en particulier en parlant d'une étoffe» (Mauron, 2001, p. 87). L'envers précise le double, la doublure. L'ombre, l'envers de mon corps, expose l'«invisible caché sous le visible» (Mauron, 2001, p. 87). Par la tache qui s'appose en surface, «le corps de l'ombre franchit un seuil léthal » (Guiomar, 1993, p. 272) en un projet de transgression extrême du seuil de la mort, car jouer sa propre mort a pour ambition d'appréhender ce passage vers l'invisible.

\section{UN «FAIRE COMME SI » DE LA MORT : RÊVE OU DON ?}

Voici qu'allongée sur le sol tumulaire, je médite sur le crâne du monde en un songe éveillé. J'effectue un retour sur moi-même et songe à l'enfant que j'étais se représentant « à l'agonie, puis à l'article de la mort, se dédoublant pour évoquer en spectateur ses derniers instants, le cérémonial qui les entoure comme ses derniers soupirs, l'envol de l'âme et la déchéance du corps, jusqu'au dernier acte [...] (Vovelle, 1983, p. 297). Ainsi couchée sur le tombeau scénique, je contemple ma propre mort. Cette douce méditation éloigne la crainte et la terreur de la mort, et favorise l'image apaisée du songe à la vie éternelle. La mort imprègne doucement la vie. Serait-ce la dernière vision du monde, le tout dernier temps? La vision du mort, en tant que support figuratif, nourrit le théâtre d'une image révélatrice d'un éventuel retour apocalyptique. La pensée de ma propre mort entre en un grand cérémonial d'un mourir qui se pose en intercesseur entre le monde des vivants et le monde des morts, qui fait don en toute humilité au cosmos des signes de sa mort jouée. Deux forces opposées s'inscrivent ici entre la vie et la mort, celle du corps blanc d'une mort sculptée prêt à vaciller et toujours retenu. Le Gisant appelle son obscur double afin que ce qui appartient à la terre lui soit rendu, que ce qui est au ciel vive. Le double est l'un des événements essentiels du passage dans l'au-delà dont l'ombre est la dernière transfiguration.

\section{MAIS, POUR QUELLE INCARNATION?}

La vision de la Mort de Soi m'amène à penser ma mort comme si elle ne cessait jamais de se porter vers l'infini; c'est ainsi que le ciel et la terre entrèrent en confusion. C'est alors que l'horizon devint insaisissable, s'enfuyant vers le futur puisqu'il appartient au temps de le faire défiler et à l'espace de le capturer. Le paysage mortuaire se donne à voir dans l'étendue d'une immensité, parcourue en tout sens par le vent libertin d'une mort jouée, celle de la pensée de ma propre mort qui souffle dans le théâtre, qui affole l'œil et égare les limites d'un horizon rendu totalement invisible et imprévisible. La chose, telle que je l'ai présentée, ne peut être vue seule, 


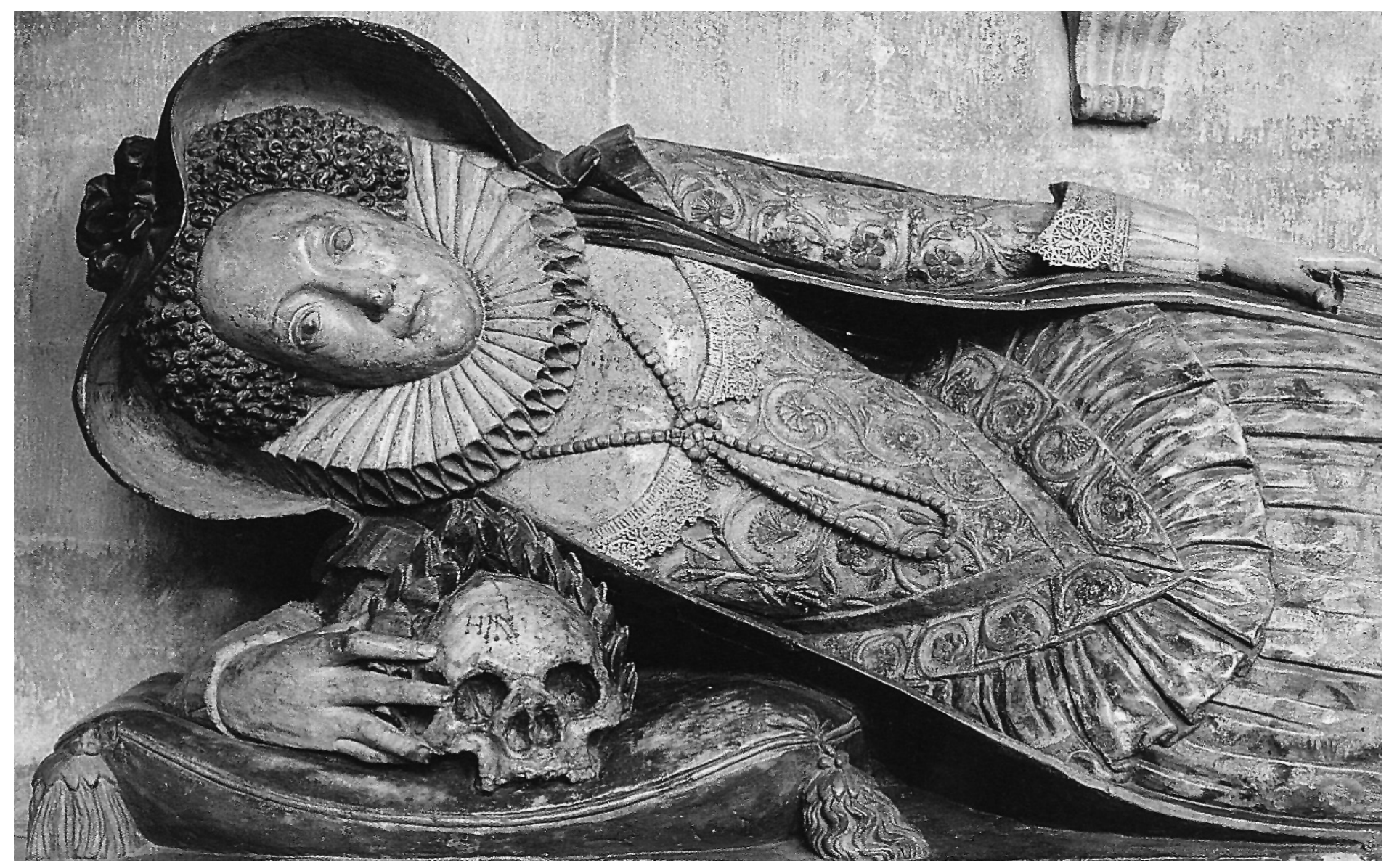

Tombe de Lady Dorothy Dodderidge (1614), cathédrale d'Exeter, Royaume-Uni.

et ne s'appréhende que par son inclusion dans un champ funéraire concrétisé par le cimetière théâtral, en tant qu'espace habité par le mort, un paysage mis en tableau sur lequel la mort s'imprime et au sein duquel elle s'exprime. Je qualifie ma mort ainsi pensée de personnage cosmique, d'allégorique absenté tissant la trame de l'univers avec les «fils lointains» qui «viennent vers lui de l'infini» (Collot, 1989, p. 47) et le relie à tous les horizons de l'espace et du temps. Il démêle les fils de son allégorie; il défait fil à fil le tapis rouge théâtral entré en confusion. La structure d'horizon telle que la définit Collot amène le dépassement du présent, et encourage l'avenir mortel à cohabiter en un ailleurs du temps. "Ils sont "apprésentés" en marge du présent vivant» (Collot, 1989, p. 48). Si l'horizon vient à se définir, il est immédiatement effacé par un futur à-venir qui propose son énigme, et un passé qui n'est plus que ressouvenir indistinct voire incommensurablement enfoui. L'horizon sur lequel je me tiens dans la pensée de ma propre mort rassemble l'espace dérobé de la nécropole dans un théâtre enclos et concourt à ouvrir la faille invisible d'un paysage, prêt d'une part à sombrer dans la profondeur du passé introuvable puisque néantisé, d'autre part à être propulsé en une échappée extati- que dans le futur inconcevable puisque inatteignable de sa propre mort. Collot qualifie ce présent d'intenable et le fait correspondre à «l'évanescence d'un pur instant, comme le hors-lieu de l'horizon ne s'ouvre qu'à partir d'un point de vue incarné» (Collot, 1989, p. 53). C'est par mon incarnation dans la mort qu'en tant que figure théâtrale je peux participer au «fond obscur des choses» (Collot, 1989, p. 28). Je me suis engagée dans l'opacité du monde; par la statuaire du Gisant et le dispositif cimetiéral qui le soutient, la pensée de ma propre mort mise en jeu fait partie du visible. Elle se pose elle-même à la vue, placée en abîme, mêlée à la corporéité d'horizon qui la fait aussi s'absenter. Elle se cache en même temps qu'elle se dévoile; elle s'abîme dans une impossibilité de présentation totale de la mort. «Le propre du visible est d'avoir une doublure d'invisible au sens strict qu'il rend présent comme une certaine absence» (Merleau-Ponty, 1964, p. 85). Mon corps costumé en Gisant est la condition qui me fait apparaître en une figure capable de délimiter l'univers en une re-présentation contemplative d'une partie de soi, mais de l'illimiter en rendant béant le chaos dans lequel je cogite. Car, au-delà de l'horizon du monde, la mort illusoire ouvre le gouffre d'une doublure pressentie, un monde de néant où il n'y a sans doute rien. Puisque le monde se renvoie l'indéfini de perspectives en perspectives, puisque le point de fuite ne cesse de reculer, refusant toute saisie par le regard, laissant la chose toujours plus éloignée. Au risque de me laisser noyer dans le gouffre de la confusion des horizons, j'ouvre la profondeur, car elle est le lieu, le site de la chute vertigineuse dans lequel le mort ancre ses pas. Par ce geste, j'envisage de faire la passe au passé, afin de prendre pied dans le présent, de poser le pied, de passer et de re-faire la passe au théâtre qui est un art du vivant afin que soit restitué le sujet vif de la pensée de ma propre mort.

\section{Bibliographie}

ARIÈS, Ph. (1985). L'Homme devant la Mort, tome 1 Le temps des gitans, Paris, Seuil.

COLLOT, M. (1989). La poésie moderne et la structure d'horizon, Paris, Presses universitaires de France.

GUIOMAR, M. (1993). Principes d'une esthétique de la Mort, Paris, Librairie José Corti.

MAURON, V. (2001). Le signe incarné, Paris, éditions Hazan.

MERLEAU-PONTY, M. (1964). L'œil et l'Esprit, Paris, Gallimard.

VOVELLE, M. (1983). La Mort et l'Occident, de 1300 à nos jours, Paris, Gallimard. 